\title{
Alimentación saludable en preescolares: un tema de interés para la salud pública
}

\section{Healthy eating in preschoolers: a topic of interest for public health}

Autores:

Carmen Elisa Abadeano Sanipatin ${ }^{1,2}$

Mariana de Jesús Mosquera Guilcapi ${ }^{1,3}$

Jenny Elizabeth Coello Viñán ${ }^{1,4}$

Byron Eduardo Coello Viñán ${ }^{1,5}$

${ }^{1}$ Escuela Superior Politécnica de Chimborazo, Riobamba-Ecuador.

${ }^{2}$ Centro de Salud tipo “A” Jatunpamba, Guamote-Ecuador.

${ }^{3}$ Centro de Salud tipo "C" Ilapo, Guano-Ecuador.

${ }^{4}$ Centro de Salud tipo B Chambo, Chambo-Ecuador.

${ }^{5}$ Centro de Salud ESPOCH Lizarzaburu, Riobamba-Ecuador.

Autor de correspondencia: Jenny Elizabeth Coello Viñán, Escuela Superior Politécnica de Chimborazo, Riobamba, Ecuador. Email: jennytutocv@yahoo.es. Teléfono: 0997869564.

\section{RESUMEN}

Diversos organismos internacionales reconocen a los problemas de desórdenes nutricionales como una problemática de salud que afecta a todo el mundo. La forma en que los seres humanos asumen su alimentación repercute en su disposición intrínseca ante las enfermedades, siendo particularmente impactante durante las etapas tempranas de la vida, sobre todo en edades preescolares por su incidencia en el desarrollo del individuo. Alrededor de esta situación, los autores del manuscrito que se presenta realizaron un análisis epidemiológico y salubrista desde diferentes posicionamientos establecidos en la bibliografía que fue consultada.

Palabras clave: trastornos de la nutrición del niño, programas de nutrición, preescolar, factores de riesgo.

\section{ABSTRACT}

Several international organizations recognize the problems of nutritional disorders as a health problem that affects the entire world. The way how human beings assume their food has an impact on their intrinsic disposition towards diseases, being particularly striking in the early stages of life, especially in pre-school age because of its impact on the development of the individual. About this situation, the authors of the manuscript carried out an epidemiological and salubrious analysis from different positions established in the reviewed literature.

Keywords: Child Nutrition Disorders; Nutrition Programs; Child, Preschool; Risk Factors. 


\section{INTRODUCCIÓN}

Criterios emitidos por la OMS señalan a los desórdenes nutricionales como la epidemia del siglo XXI; la que fundamentalmente se manifiesta en la población infantil, dejando secuelas que suelen durar toda la vida. ${ }^{(1)}$

A nivel mundial, se calcula que 293 millones de niños menores de cinco años padecen de anemia, representando un aproximado del $47 \%$ de infantes que viven en países subdesarrollados. ${ }^{(2)} \mathrm{Al}$ respecto, Páez Valery et al. ${ }^{(3)}$ se refieren a la falta de micronutrientes como "hambre oculta"; la que afecta a una cifra superior a 2 billones de personas en el mundo, en una proporción aproximada de 1 por cada 3 habitantes, especialmente concentrada en infantes, recién nacidos y gestantes.

Como ciencia, la nutrición humana se encarga del estudio de la relación entre los alimentos y los seres vivos que los consumen. En el caso de los humanos, esta se concentra en el análisis de esas necesidades y las enfermedades que las inadecuadas prácticas al respecto pueden producir; además, de establecer parámetros de calidad de los elementos que componen las dietas de los individuos en los diferentes entornos sociales y naturales. ${ }^{(4-6)}$

Una dieta adecuada puede prevenir o mejorar determinadas enfermedades. En el ser humano, el normal funcionamiento del cuerpo requiere de la apropiada nutrición a nivel celular; por lo que, las buenas prácticas al respecto resultan un factor primordial para el vigor y el desempeño de las personas. ${ }^{(4,7,8)}$

Uno de los desórdenes nutricionales de mayor prevalencia reconocidos a nivel mundial resulta la anemia, especialmente en niños menores de 5 años independientemente del desarrollo socio-económico de los países que aportan los respectivos datos. ${ }^{(9)}$

La malnutrición resulta un problema a nivel mundial; se calcula que 150 millones de niños de 1 a 10 años sufren de esta problemática; Al respecto, entre los menores de 5 años se estima que son 4 millones, cifra que sufrió un incremento del 7\%. ${ }^{(10)}$

Para Behm Rosas, ${ }^{(11)}$ la hambruna constituye una de las principales causas de mortalidad en América Latina; problemática que continúa sin solución, aunque existen muestras de crecimiento socioeconómico en la región. Al respecto, el Programa Mundial de Alimentos de Naciones Unidas, reportó que en esa región existen alrededor de 9 millones de niños menores de 5 años que padecen desnutrición, y que una cifra similar están en alto riesgo de afectarse debido a los niveles de pobreza en determinadas comunidades. ${ }^{(12)}$

En muchos países de América Latina, aún persisten elevados índices de desigualdades socioeconómicas; las que repercuten en los estados nutricionales de sus poblaciones, especialmente en niños preescolares; comúnmente, las costumbres alimenticias suelen ser monótonas y deficientes en alimentos ricos en hierro, cinc, calcio, tiamina, riboflavina, folatos, piridoxina, vitamina C y vitamina A. ${ }^{(13,14)}$ 
Se calcula que unos 129 millones de niños menores de cinco años en esos países poseen un peso y talla inferior al recomendable, lo que indica malnutrición; al respecto, destacan algunos países andinos como: Ecuador, Bolivia y Perú, en lo que llega a ser hasta de cuatro a cinco veces superior la presencia del bajo peso. ${ }^{(15)}$

Pajuelo Ramírez y Miranda Cuadros ${ }^{(10)}$ plantean que, entre las manifestaciones de desorden nutricional de mayor presencia en los niños peruanos menores de 5 años están la desnutrición crónica, la obesidad y la deficiencia de micronutrientes. Esa situación que requiere de la toma de acciones integrales en las comunidades.

Longhi et al. ${ }^{(16)}$ concluyeron que la desnutrición infantil es uno de los problemas de salud de mayor relevancia en la actualidad de algunas regiones de Argentina. Los hallazgos mostraron que esa patología ha descendido en ese contexto, sin disminuir hasta los niveles deseables de Argentina, influyendo de manera directa en las tasas de morbilidad y mortalidad, así como en la prevalencia de determinadas enfermedades crónicas no transmisibles.

La relación existente entre alimentación inadecuada y aparición de enfermedades prevenibles ha hecho que el tema de la búsqueda de la alimentación saludable constituya una prioridad para la salud pública. ${ }^{(17,18)}$

Según Behm Rosas, ${ }^{(11)}$ en su análisis sobre los determinantes económicos y sociales de la mortalidad en América Latina, la desnutrición continúa siendo no resuelta en América Latina. Este autor considera que el crecimiento económico de la región no ha incidido en esta situación inherente a la salud pública que tiene mayor presencia en la población infantil.

En Ecuador, las medidas establecidas en Plan Nacional del Buen Vivir 2013-2017 tuvieron un impacto positivo en la disminución de la desnutrición crónica; en relación con lo anterior; en 1986, la cifra era del 40.2\%, mientras que para 2012 se redujo al 25.3\%. Sin embargo, este continúa siendo una problemática de salud no resuelta que amerita el desarrollo de las acciones correspondientes. $^{(19)}$

\section{DESARROLLO}

Según los criterios de Robalino Flores et al., ${ }^{(20)}$ las comunidades de la región andina ecuatoriana resultan altamente afectadas por la desnutrición, debido a la accesibilidad a los recursos y la educación; situación que afecta especialmente a los niños. Esas autoras también consideran que, esta resulta una temática insuficientemente investigada en esa nación.

Los autores de una investigación, relativa a la desnutrición en pacientes pediátricos hospitalizados en una institución ecuatoriana, reportaron una tasa de ese indicador del $37,1 \%$, cifra que resultó significativa en ese contexto. Ellos recomiendan que esa patología debiese ser considerada en este tipo de paciente que ingresa en una casa de salud, independientemente de su causa de internamiento. ${ }^{(21)}$ 
A partir de los resultados de la Encuesta Nacional de Salud y Nutrición (ENSANUT), aplicada en Ecuador entre 2011 y 2013, el Ministerio de Inclusión Económica y Social (MIES) implementó una estrategia de entrega de suplementos con micronutrientes (fundamentalmente hierro) a más de 350000 preescolares, que formaba parte del programa Creciendo con Nuestros Hijos (CHN), en 2200 Centros Infantiles del Buen Vivir (CIV). ${ }^{(22)}$

Las acciones preventivas que se implementen en relación con la nutrición deben ser simultáneas y sinérgicas con participación comunitaria, de manera que eviten llegar a la enfermedad. Este tipo de estrategia salubrista plantea diferentes desafíos que son abordados desde diferentes sus $\operatorname{aristas}^{(8)}$

Al respecto, Vickers ${ }^{(23)}$ plantea que los primeros años de vida constituyen etapas críticas del desarrollo, en las que alimentación juega un importante papel en la prevención de enfermedades.

Las enfermedades ligadas a la malnutrición continúan siendo un importante problema de salud que afecta a los niños a nivel mundial. Estas afectan principalmente a aquellos pacientes pediátricos con factores genéticos, metabólicos, psicosociales y ambientales. Los malos hábitos en la alimentación, además de la indiferencia y falta de percepción de los padres, resultan un elementos de riesgo al respecto, lo que indica una necesaria promoción de conocimientos sobre nutrición saludable. ${ }^{(15,24)}$

Ningún alimento en particular posee el suficiente valor nutritivo, para garantizar de forma aislada el balance de nutrientes que requiere el ser humano para obtener energía metabólicamente utilizable. Al respecto, resulta recomendable incorporar una dieta balanceada que incorpore cereales, verduras y frutas, grasas y aceites, carne, pescado, leche, huevos, leguminosas y otros; de manera que proporcione la cantidad y calidad de las sustancias requeridas para el funcionamiento adecuado del organismo. ${ }^{(25)}$

Durante la etapa preescolar, la alimentación constituye una determinante social que desarrolla un importante papel para el equilibrio físico y mental del niño; debido a que en este momento se forman los hábitos alimenticios, los que definirán el futuro estado nutricional; fase en la cual los padres tienen el rol fundamental de proveer y educar a sus hijos sobre esta temática. ${ }^{(26)}$

Las edades comprendidas entre los 3 y 5 años marcan el desarrollo cognitivo; ya que, desde ese momento, el niño se encamina hacia su dominio y crea las bases del pensamiento lógico. ${ }^{(15)}$

El aporte que la alimentación saludable proporciona al crecimiento y desarrollo del niño se aprecia desde su formación intrauterina la que en la etapa preescolar asume una significación preponderante al favorecer el desarrollo fisco mental y social de este. Autores como Moreno-Villares ${ }^{(25)}$ plantean que durante los dos primeros años de vida existe un periodo de crecimiento acelerado en el niño; que al entrar en la edad preescolar (3-5 años) esta característica se estabiliza y el individuo comienza a crecer a un ritmo promedio de 6-8 cm/año, ganando entre de $2-3 \mathrm{~kg} / \mathrm{año}$. 
Una dieta adecuada puede prevenir o mejorar determinadas enfermedades en el ser humano, el normal funcionamiento del organismo requiere de la apropiada nutrición a nivel celular; por lo que, las buenas prácticas al respecto resultan un factor primordial para el vigor y el desempeño de las personas. ${ }^{(7)}$

La edad preescolar se reconoce como una etapa crítica, en la que se pueden obtener los máximos beneficios de la promoción de la salud y prevención de enfermedades. Al respecto, la realización de acciones dirigidas hacia la infancia temprana en pro de un desarrollo saludable resulta especialmente necesarias y efectivas. Estas deben incluir la búsqueda de una alimentación adecuada y la realización de actividad física en ese grupo poblacional; por lo que padres y tutores legales deben estar apropiadamente educados en esos particulares. ${ }^{(27)}$

En Ecuador, más de un cuarto de los niños en edad preescolar sufre de anemia; y una cifra muy similar está reportada con baja talla. ${ }^{(28)}$ En agosto de 2018, las cifras relativas a la desnutrición ecuatorianas colocaron a la provincia Chimborazo como la sexta con mayor presencia de esta problemática de salud (71 casos). ${ }^{(29)}$

Los trastornos nutricionales en la etapa preescolar continúan siendo un problema de salud cuya solución está pendiente, a nivel mundial, regional y local. ${ }^{(15,17,30)}$

Los hábitos de vida saludables deben ser desarrollados en el individuo desde edades tempranas, lo que resulta recomendable alcanzar mediante el desarrollo de programas de prevención y educación; siendo esta la más efectiva herramienta en la reducción de la incidencia de patologías relacionadas con alimentación excesiva o dietas poco equilibradas. ${ }^{(25)}$ Algunos autores plantean que este resulta un problema marcado por algunos determinantes de carácter socioeconómico y educativo; lo que da lugar a la necesidad de intervenciones de promoción desde un enfoque comunitario y participativo. ${ }^{(17)}$

Una de las vías instrumentadas por la ciencia para contribuir a la mitigación de la desnutrición como flagelo humano lo constituye la aplicación de intervenciones educativas dirigidas a la promoción de la alimentación saludable en la etapa prescolar; mediante la educación de las madres responsables de esta tarea a nivel de la estructura familiar. ${ }^{(31)}$

Evidencia científica consultada indica la existencia de una contradicción en cuanto a la conducta que los padres deben tomar frente a la formación de hábitos alimenticios en sus hijos. Al respecto, se ha podido comprobar que las estrategias de elogios, recompensas y coercitivas no resultan efectivas en los niños. ${ }^{(32,33)}$

La infancia constituye una etapa trascendental dentro del proceso de desarrollo del hombre, que se caracteriza por formación de las bases biológicas, psicológicas y sociales que marcarán al individuo por el resto de su vida. Este es un momento de la vida del individuo en que la nutrición tiene un rol preponderante si se quieren alcanzar los niveles deseables con respecto a esas tres dimensiones. ${ }^{(34)}$ 
El contexto impacta directamente en el desarrollo del infante preescolar, en particular a la familia, la que debe trabajar en función de su bienestar integral para que adquiera hábitos nutricionales que incidirán en él por el resto de su vida. ${ }^{(25)}$ En este período, los niños empiezan a asumir el control de sí mismos y adecuar su ambiente según sus necesidades. ${ }^{(35)}$ La postura ante la alimentación no escapa a esos cambios conductuales, comienzan a mostrar interés por algunos alimentos, volviéndose caprichosos en ocasiones y manifestándose con poco apetito. Los padres y cuidadores tendrán que incidir en este sentido para que adopte costumbres saludables. ${ }^{(25)}$

La UNICEF(24) define la desnutrición crónica como el retraso del crecimiento del niño al no alcanzar la talla esperada según su edad. Esta patología ha sido destacada como una emergencia silenciosa en la población infantil; ya que no se detecta inmediatamente que comienza a afectar y acarrea consecuencias graves que se manifestarán a lo largo de toda la vida de la persona.

La antropometría constituye el principal método de diagnóstico de la desnutrición en los pacientes pediátricos; al respecto, Lázaro-Cuesta et al ${ }^{(36)}$ recomiendan el uso adicional de técnicas de laboratorio, de manera que permita determinar la presencia de anemia, así como los niveles de vitamina A, hierro, el zinc y otros micronutrientes.

Según Reyes Moralesa et al. ${ }^{(27)}$ existe abundante evidencia de que las intervenciones encaminadas a la promoción de prácticas saludables en los niños desde edades tempranas, que pueden realizarse mediante la capacitación de los padres, para que estos se conviertan en formadores de estilos de vida adecuados que alcancen mayor permanencia. Al respecto, varios estudios muestran modificación de conductas favorables. ${ }^{(37,38)}$

Aquino Rojas ${ }^{(34)}$ desarrolló un proceso investigativo en el que aplicó una intervención educativa sobre desnutrición, dirigida madres de niños menores de seis meses, a partir del cual concluyó que las actividades realizadas lograron elevar significativamente el nivel de conocimiento en muestra de estudio.

Los niños desnutridos tienen un riesgo mayor de morir durante la infancia; su desarrollo físico e intelectual se ve limitado, al igual que su capacidad de aprender y trabajar al convertirse en adulto, por lo que disminuyen las oportunidades de desarrollo económico y profesional. ${ }^{(39,40)}$

Lázaro Cuesta et al. ${ }^{(36)}$ consideran que el diagnóstico de desnutrición en los niños no debe establecerse únicamente, atendiendo a criterios relacionados con la antropometría; esos autores parten de que la deficiencia de vitamina A, hierro y el zinc, además de otros micronutrientes constituyen la principal causa de la anemia y que sería recomendable incluir otros procedimientos de laboratorio.

Ballonga Paretas et al. ${ }^{(41)}$ plantean que la adecuada nutrición en la infancia debe incluir minerales esenciales y vitaminas que necesita el organismo. La carencia de esos elementos produce una insuficiencia en la alimentación que genera fatiga, limitación en la capacidad de aprendizaje, problemas de inmunidad, entre otras consecuencias. 
Algunos autores afirman que la desnutrición infantil implica un alto costo económico y social reflejados en gastos hospitalarios, de educación, disminución de la capacidad laboral y productividad, etc.); por lo que recomiendan trabajar en la prevención de esa enfermedad y en la promoción de salud al respecto. ${ }^{(16)}$

Fundamentándose en criterios establecidos por la UNICEF, Osorio et al. ${ }^{(42)}$ establece los siguientes factores que inciden sobre la desnutrición infantil en diferentes niveles de análisis:

\section{Comunitario}

- Características del contexto socioeconómico y cultural.

- Eficiencia de las políticas y programas de salud.

- Gobernanza y participación social de los actores en la toma de decisiones.

- Calidad de los servicios sanitarios y la higiene comunitaria.

- Acceso al agua potable y los alimentos.

- Existencia de espacios acondicionados para el deporte y la recreación.

- Localización geográfica de la comunidad.

\section{Familiar}

- Uso y acceso a los servicios del sistema de salud.

- Calidad de vida colectiva e individual (incluye la alimentación).

- Características físicas, sociales y psicológicas del núcleo familiar.

- Clase social en la que se insertan.

- Ingresos per cápita.

- Etnia a la que pertenece.

\section{Madre}

- Estado de salud general.

- Experiencia en el cuidado infantil.

- Conductas preventivas conscientes.

- Autonomía económica y escolarización.

- Número de hijos.

\section{Niño}

- Edad y sexo.

- Peso y Apgar al nacer.

- Elementos hereditarios. 
Actualmente, las estrategias dirigidas a la malnutrición son cada vez más complejas debido al número de factores de riesgo a tener en cuenta y la variedad de manifestaciones que se presentan; la coexistencia de anemia, talla baja, sobrepeso u obesidad en una misma comunidad, plantea riesgos diferenciados que deben ser afrontados en base a sus particularidades y su potencialidad de causar enfermedad a corto o largo plazo; aunque la indicación de una alimentación saludable adecuadamente balanceada resulta denominador común para la mayoría de los casos. ${ }^{(43)}$

Longhi.et al. ${ }^{(16)}$ consideran que la desnutrición infantil conlleva un costo económico y social alto; los que se reflejan a través de los gastos hospitalarios, educación, poca capacidad laboral y productividad, entre otros.). La recomendación que realizan al respecto es la de dirigir los esfuerzos y recursos a la prevención de esa enfermedad.

En relación con lo anterior, la experiencia peruana del marco del "Plan Nacional para la Reducción de la Anemia 2017-2021" se proponen reducir significativamente la anemia entre niños menores de 5 años; el plan de acción se centra en cinco acciones principales: ${ }^{(44)}$

- Administrar suplementos de minerales y vitaminas en niños menores de 24 meses y embarazadas para prevenir y tratar la anemia.

- Generar prácticas de alimentación integral mediante la educación, para inculcar el consumo de elementos ricos en hierro y otros nutrientes en la adecuada cantidad.

- Implementar la intersectorialidad durante el desarrollo de intervenciones comunitarias dirigidas a la generación de conductas nutricionales saludables y la mejora del acceso a los alimentos esenciales en los hogares rurales.

- Establecer un mecanismo de seguimiento y control del impacto de las intervenciones al respecto.

- Priorizar las zonas geográficas críticas, favoreciendo la producción y comercialización de alimentos ricos en micronutrientes destinados a la alimentación de los diferentes sectores de la población necesitada.

La intervención educativa es reconocida como una de las acciones de mayor eficiencia en la prevención de la desnutrición y la promoción de conductas saludables al respecto. ${ }^{(31)}$ Algunos autores la definen como la implementación de una serie de actividades planificadas a partir de un diagnóstico de la problemática de salud en cuestión; la misma tiene como objetivo producir un cambio en el conocimiento sobre objeto de estudio en cuestión y la conducta de los individuos a quienes va dirigida. Se recomienda realizarla desde un enfoque familiar y comunitario para el alcance de su propósito en el menor plazo posible. ${ }^{(45)}$

Mundialmente, se reportan experiencias en las que se muestran resultados positivos de las intervenciones educativas dirigidas a la prevención de la anemia y sus consecuentes afectaciones del desarrollo físico y mental en los pacientes pediátricos. ${ }^{(28)}$

En el mundo, la ferropenia constituye la causa más frecuente de anemia en niños, especialmente en los preescolares. Algunos países sudamericanos desarrollan programas encaminados a la prevención y tratamiento de esa problemática de salud; los que ya muestran resultados positivos. ${ }^{(44)}$ 
Algunos autores consideran que actualmente, América Latina atraviesa una etapa de transición nutricional; en este contexto coexisten diferentes manifestaciones al respecto: desnutrición, sobrepeso, obesidad y carencias de micronutrientes; hasta hace muy pocos años solo se hablaba de la primera de estas. Un ejemplo de lo mencionado es el caso de Argentina, donde diferentes estudios arrojan que, al menos 3/10 niños y adolescentes padecen de exceso de peso. ${ }^{(36)}$

Miranda et al. ${ }^{(46)}$ informan que el Programa Multisectorial Desnutrición Cero en Bolivia, desarrollado por el Consejo Nacional de Alimentación y Nutrición de esa nación, no ha logrado disminuir la prevalencia de la anemia en las poblaciones vulnerables a pesar de las acciones.

En 2013, un estudio realizado en Medellín persiguió el objetivo de evaluar la efectividad de la fortificación dietética empleando un polvo rico en micronutrientes en 90 preescolares sanos, durante nueve semanas. Los investigadores concluyeron que, aunque los suplementos fueron bien asimilados por los infantes de la muestra, sus niveles hematológicos y estado nutricional no mejoraron; lo que indicó que resultan innecesarios en los casos que no poseen esa defíciencia alimentaria. $^{(15)}$

Más del 25\% de los preescolares ecuatorianos sufren de anemia; y una cifra semejante está informada como baja talla. ${ }^{(28)}$ Según la Dirección Nacional de Vigilancia Epidemiológica (2018), durante la semana 32 del 2018, los datos relacionados con a la desnutrición arrojan que la provincia Chimborazo resulta la sexta más afectada por este problema de salud (71 casos). Autores como Calderón-Vallejo ${ }^{(47)}$ que sería recomendable con la realización de intervenciones nutricionales en pacientes pediátricos.

Aunque los resultados respecto al alcance del objetivo final de eliminar desnutrición severa, se pueden mencionar varias acciones del estado ecuatoriano dirigidas en ese sentido, tales como: fortificación alimentaria en niños, programa "Nutri bebé" que distribuyó alimentos complementarios, micronutrientes, vitamina A y chispitas nutricionales, además de la creación de las Unidades Nutricionales Integrales. ${ }^{(48)}$

En Ecuador, en 2011, el Ministerio de Salud Pública, llevó a cabo un programa de administración de suplementos de micronutrientes al que denominó "Chis-paz", el que estuvo compuesto por hierro, ácido fólico, zinc, vitamina A, vitamina C; el antecedente de esta acción data de 1997 cuando se realizó una campaña de distribución de vitamina A (aunque no existen datos concretos de su efecto). ${ }^{(28)}$

Los individuos en edad preescolar ( 2 a 5 años) se encuentran en una etapa denominada preoperacional; que es la segunda en importancia para el desarrollo cognitivo, en este momento se dirige hacia su dominio y sienta bases del pensamiento lógico. ${ }^{(15)}$ En este punto, la alimentación tiene un papel primordial en el desarrollo mental y físico del infante; aquí se originan los hábitos alimentarios que le definirán su estado nutricional futuro; por lo que, el papel educador y proveedor de los padres resulta fundamental. ${ }^{(26)}$ 
Entre los 3 y 5 años, el niño comienza a estabilizar su crecimiento a un ritmo promedio de 6-8 $\mathrm{cm} /$ año y ganan de $2-3 \mathrm{~kg} /$ año. ${ }^{(25)}$ Los niños que se desnutren se exponen a mayores riesgos de muerte durante la infancia, se limita su potencial desarrollo físico e intelectual y se restringe su capacidad de aprender y trabajar en la adultez, por lo cual se limitan así las oportunidades de desarrollo profesional y económico. ${ }^{(39,40)}$

Chuquimarca-Chuquimar et al. ${ }^{(28)}$ concluyen que la administración de suplementos ricos en micronutrientes actúa directa y positivamente sobre la mejoría en el nivel de anemia, así como en el del indicador talla/edad de los preescolares, pero que sería pertinente implementar acciones para modificar conductas alimentarias en las comunidades donde se detecta esa problemática de salud.

La anemia constituye una enfermedad que se produce cuando los niveles de concentración de hemoglobina y el número de glóbulos rojos se encuentran por debajo de lo normal, atendiendo a distintos factores como: género, edad y área geográfica de residencia. Esta patología es un indicador indirecto de insuficiencia de hierro y otros micronutrientes. ${ }^{(2,49)}$

\section{CONCLUSIONES}

Los desórdenes nutricionales son reconocidos como la epidemia del siglo XXI; al respecto, la población infantil resulta una de más afectadas con consecuencias impredecibles en los casos de niños en edad preescolar.

Aunque el estado ecuatoriano desarrolla un conjunto de acciones desde 2013 fundamentalmente, la desnutrición infantil continúa siendo un problema de salud en ese país.

Diferentes autores plantean que la solución de esa problemática de salud debe ser implementada en el sistema primario de atención, a partir de un conjunto de acciones integradoras dirigidas a la mitigación o erradicación de los factores de riesgo relacionados con los desórdenes nutricionales.

Conflicto de intereses: los autores declaran que no existen.

Declaración de contribuciones: Carmen Elisa Abadeano Sanipatin, Mariana de Jesús Mosquera Guilcapi, Jenny Elizabeth Coello Viñán y Byron Eduardo Coello Viñán trabajaron en la búsqueda bibliográfica, recopilación, procesamiento e interpretación de la información; así como en la redacción del artículo.

\section{REFERENCIAS BIBLIOGRÁFICAS}

1. Nobre LN, Lessa AC, Oliveira HC, Lamounier JA, Francischini SC. Iron-deficiency anemia and associated factors among preschool children in Diamantina, Minas Gerais, Brazil. Rev Nutr [Internet]. 2017 Mar [citado 2018 Jul 29]; 30(2): 185-196. Disponible en: http://www.scielo.br/scielo.php?script=sci_arttext\&pid=S1415-52732017000200185\&lng=en. http://dx.doi.org/10.1590/1678-98652017000200004. 
2. Núñez-Arboleda MI, Fonseca-Díaz GM. Guía educativa sobre la anemia ferropénica para disminuir su aparición en niños menores de cinco años en el Hospital General Riobamba [Tesis en opción al grado de Licenciada en Enfermería]. Ambato: Uniandes; 2017 [citado 2018 Ago 08]. Disponible en: http://186.3.45.37/bitstream/123456789/6039/1/PIUAENF011-2017.pdf.

3. Páez-Valery MC, Naddaf G, Barrado D, Cioccia AM, Hevia P. Prohepcidina y estado del hierro e inflamación en escolares sanos o H. pylori positivos asintomáticos. Acta bioquím. clín. latinoam. [Internet]. 2017 Jun [citado 2018 Jul 26]; 51(2): 183-194. Disponible en: h t t p : / / w w w. scielo.org.ar/scielo.php? script=sci_arttext\&pi$\mathrm{d}=\mathrm{S} 0325-29572017000200003 \& \operatorname{lng}=\mathrm{es}$.

4. Izquierdo-Hernández A, Armenteros-Borrell M, Lancés-Cotilla L, Martín-González I. Alimentación saludable. Rev Cubana Enfermer [Internet]. 2004 Abr [citado 2018 Ago 09]; 20(1): 1-1. Disponible en: http://scielo.sld.cu/scielo.php?script=sci_arttext\&pi$\mathrm{d}=$ S0864-03192004000100012\&lng=es.

5. Daniels LA, Mallan KM, Battistutta D, Nicholson JM, Perry R, Magarey A. Evaluation of an intervention to promote protective infant feeding practices to prevent childhood obesity: outcomes of the NOURISH RCT at 14 months of age and 6 months post the first of two intervention modules. Int J Obes (Lond) [Internet]. 2012 [citado 2018 Ago 07]; 36(10): 1292-8. Disponible en: https://www.nature.com/articles/ijo201296. doi: 10.1038/ijo.2012.96.

6. Savage JS, Birch LL, Marini M, Anzman-Frasca S, Paul IM. Effect of the INSIGHT Responsive Parenting Intervention on Rapid Infant Weight Gain and Overweight Status at Age 1 Year: A Randomized Clinical Trial. JAMA Pediatr [Internet]. 2016 [citado 2018 Ago 11]; 170(8): 742-9. Disponible en: https://jamanetwork.com/journals/jamapediatrics/fullarticle/2527086. doi: 10.1001/jamapediatrics.2016.0445.

7. Milte CM, Thorpe MG, Crawford D, Ball K, McNaughton SA. Associations of diet quality with health-related quality of life in older Australian men and women. Experim Gerontol [Internet]. 2015 [citado 2018 Ago 13]; 64: 8-16. Disponible en: https://www.sciencedirect.com/science/article/pii/S0531556515000601?via\%3Dihub. DOI: https://doi.org/10.1016/j.exger.2015.01.047.

8. França-Tarragó O, Crestanello F, Müller A, Silveri A, Pons JE. El problema del sobrepeso y la obesidad en la niñez y adolescencia. Una mirada desde la Bioética. Anfamed [Internet]. 2017 Jul [citado 2018 Jun 01]; 4(1): 14-71. Disponible en: http://www.scielo.edu.uy/scielo.php?scrip$\mathrm{t}=$ sci_arttext\&pid=S2301-12542017000100014\&lng=es. $\quad$ http://dx.doi.org/10.25184/anfamed2017.4.1.4.

9. Milman N. Fisiopatología e impacto de la deficiencia de hierro y la anemia en las mujeres gestantes y en los recién nacidos/infantes. Rev peru ginecol obstet [Internet]. 2012 [citado 2018 Jul 19]; 58(4): 293-312. Disponible en: http://www.scielo.org.pe/scielo.php?script=sci_arttext\&pid=S2304-51322012000400009\&lng=es. 
10. Pajuelo-Ramírez J, Miranda-Cuadros M. La coexistencia de problemas nutricionales en niños menores de 5 años en el Perú 2007-2010. An Fac med [Internet]. 2016 Oct [citado 2018 Ago 21]; 77(4): 345-349. Disponible en: http://www.scielo.org.pe/scielo.php?script=sci_arttext\&pid=S1025-55832016000400005\&lng=es.

11. Behm-Rosas H. Determinantes económicos y sociales de la mortalidad en América Latina. Rev Cubana Sal Púb [Internet]. 2017 [citado 2018 Ago 17]; 43(2). Disponible en: http://www.revsaludpublica.sld.cu/index.php/spu/article/view/939/870.

12. Organización de las Naciones Unidas para la Alimentación y la Agricultura. Panorama de la inseguridad alimentaria en América Latina y el Caribe. Roma: FAO; 2015 [citado 2018 Ago 30]. Disponible en: http://www.fao.org/3/a-i4636s.pdf.

13. Hijar G, Aramburu A, Hurtado Y, Suárez V. Fortificación del arroz para corregir la deficiencia de micronutrientes en niños de 6 a 59 meses de edad. Rev Panam Salud Publica [Internet]. 2015 [citado 2018 Sep 01]; 37(1): 52-58. Disponible en: https://www.scielosp.org/pdf/rpsp/2015.v37n1/52-58/es.

14. Petkovic J, Jull J, Armstrong R, Boyer Y, Cuervo LG, et al. Protocol for the development of a CONSORT-equity guideline to improve reporting of health equity in randomized trials. Implementation Science [Internet]. 2015 [2018 Ago 11]; 10(46): 10-21. Disponible en: https://implementationscience.biomedcentral.com/articles/10.1186/s13012-015-0332-z. DOI: http://dx.doi.org/10.1186/s13012-015-0332-z.

15. Orozco J, Ruiz A. Efectividad de un Programa Educativo para mejorar Conocimientos sobre Alimentación Saludable en los niños y niñas de 5 años de una I.E.I. de Chiclayo-2013. Rev Sal \& Vida Sipanense [Internet]. 2014 [citado 2018 Sep 25]; 1(1). Disponible en: http://revistas.uss.edu.pe/index.php/SVS/article/view/68/67.

16. Longhi F, Gómez A, Zapata ME, Paolasso P, Olmos O, Ramos-Margarido S. La desnutrición en la niñez argentina en los primeros años del siglo XXI: un abordaje cuantitativo. Salud Colectiva [Internet]. 2018 [citado 2018 Ago 21], 14(1): 33-50. Disponible en: http://revistas.unla.edu.ar/saludcolectiva/article/view/1176/pdf. https://doi.org/10.18294/sc.2018.1176.

17. González-Rodríguez A, García-Padilla FM, Martos-Cerezuela I, Silvano-Arranz A, Fernández-Lao I. Proyecto ANDALIES: consumo, oferta y promoción de la alimentación saludable en los centros de educación secundaria de Andalucía. Nutr Hospit [Internet]. 2015 [citado 2018 Sep 01]; 31(4): 1853-1862. Disponible en: http://www.nutricionhospitalaria.com/pdf/8450.pdf. https://dx.doi.org/10.3305/nh.2015.31.4.8450.

18. Willett W. Nutritional Epidemiology. New York: Oxford University Press; 2013.

19. Fernandez-Velez Y, Caballero-Torres A, Mederos-Mollineda K. Evaluación Nutricional en preescolares atendidos en el Hospital de Manglaralto, Provincia Santa Elena. Ciencia Unemi [Internet]. 2015 [citado 2018 Ago 24]; 8(15): 103-108. Disponible en: http://ojs.unemi.edu.ec/index.php/cienciaunemi/article/view/204/202. doi: http://dx.doi.org/10.29076/issn.2528-7737vol8iss15.2015pp103-108p. 
20. Robalino-Flores XR, Balladares-Saltos AM, Guerendiain-Margni ME, Morales-Marín F. Anthropometric and hematological tests to diagnose nutritional deficiencies in schoolchildren of indigenous communities living in the Andean region of Ecuador. Rev Nutr [Internet]. 2017 Dic [citado 2018 Sep 10]; 30(6): 723-733. Disponible en: http://www.scielo.br/scielo.php?script=sci_arttext\&pid=S1415-52732017000600723\&lng=en.

http://dx.doi.org/10.1590/1678-98652017000600005.

21. Gallegos-Espinosa S, Nicolalde-Cifuentes M, Santana-Porbén S. Grupo Ecuatoriano para el estudio de la malnutrición hospitalaria. Estado de malnutrición en hospitales de Ecuador. Nutr Hospit [Internet]. 2014 [citado 2018 Sep 01]; 30(2): 425-35. Disponible en: http://www.aulamedica.es/nh/pdf/7559.pdf. https://doi.org/10.3305/nh.2014.30.2.7559.

22. Freire WB, Ramírez MJ, Belmont P, Mendieta MJ, Silva MK, Romero N, et al. Resumen Ejecutivo. Tomo I. Encuesta Nacional de Salud y Nutrición ENSANUT ECU 2011-2013. INEC/MSP: Quito; 2013.

23. Vickers MH. Early life nutrition, epigenetics and programming of later disease. Nutrients [Internet]. 2014 [citado 2018 Ago 27]; 6: 2165-78. Disponible en: https://www.mdpi.com/2072-6643/6/6/2165/htm. DOI:10.3390/nu6062165.

24. UNICEF. La niñez y adolescencia [Internet]. Lima: UNICEF; 2014. Disponible en: http://www.unicef.org/peru/spanish/children.

25. Moreno-Villares JM, Galiano-Segovia MJ. Alimentación del niño preescolar, escolar y adolescente. Pediatr integr [Internet]. 2015 [citado 2018 Sep 11]; XIX(4): 268-276. Disponible en: h t t p://www.pediatriaintegral.es/wp - content/uploads/2015/07/Pe diatria-Integral-XIX-4_WEB.pdf\#page=45.

26. Crovetto M, Henríquez C, Parraguez R, Silva-Montenegro M.J. Relación entre la alimentación institucional de Jardines Infantiles y del Hogar con el estado nutricional de los preescolares que asisten a dos Jardines Infantiles en Valparaíso, Chile. Rev Esp Nutr Hum Diet [Internet]. 2016 Mar [citado 2018 Jul 30]; 20(1): 4-15. Disponible en: http://scielo.isciii.es/scielo.php?script=sci_arttext\&pid=S2174-51452016000100002\&lng=es. http://dx.doi.org/10.14306/renhyd.21.1.175.

27. Reyes-Morales H, González-Unzaga MA, Jiménez-Aguilar A, Uribe-Carvajal R. Efecto de una intervención basada en guarderías para reducir conductas de riesgo de obesidad en niños preescolares. Bol Med Hosp Infant Mex [revista en la Internet]. 2016 Abr [citado 2018 Dic 12]; 73(2): 75-83. Disponible en: http://www.scielo.org.mx/scielo.php?script=sci_arttext\&pi$\mathrm{d}=$ S1665-11462016000200075\&lng=es. http://dx.doi.org/10.1016/j.bmhimx.2015.11.010.

28. Chuquimarca-Chuquimar R, Caicedo-Hinojosa L, Zambrano-Dolver J. Efecto del suplemento de micronutrientes en el estado nutricional y anemia en niños, Los Ríos-Ecuador; 2014-2015. MULTIMED [Internet]. 2018 [citado 2018 Dic 15]; 21(6): [aprox. 0 p.]. Disponible en: http://www.revmultimed.sld.cu/index.php/mtm/article/view/661. 
29. Dirección Nacional de Vigilancia Epidemiológica. Desnutrición cero, Ecuador, semana epidemiológica 32/2018. Quito: MSP; 2018. Disponible en: https://www.salud.gob.ec/wp-content/uploads/2016/09/GACETA-DESNUTRICI\%C3\%93N-SE-32_2018.pdf.

30. Congacha-Aushay IR. Diseño de estrategia de intervención educativa sobre alimentación saludable en madres de niños menores de 5 años. Comunidad San Pedro de Ayacón, enero a junio 2016 [Tesis en opción al grado de Especialista en Medicina Familiar y Comunitaria]. Riobamba: Escuela Superior Politécnica de Chimborazo; 2016.

31. Selva-Suárez LN, Ochoa-Alonso AA. Acciones para la prevención y control de la anemia por deficiencia de hierro en niños hasta cinco años. Rev Cubana Salud Pública [Internet]. 2011 Sep [citado 2018 Jul 29]; 37(3): 200-206. Disponible en: http://scielo.sld.cu/scielo.php?scrip$\mathrm{t}=$ sci_arttext\&pid=S0864-34662011000300003\&lng=es.

32. Kyung E. Rhee EJ, Boutelle K, Dickstein S, Seifer R, Wing R. Warm Parenting Associated with Decreasing or Stable Child BMI during Treatment. Childhood Obesity [Internet]. 2016 Abr [citado 2018 Ago 28]; 12(2). Disponible en: https://www.liebertpub.com/doi/abs/10.1089/chi.2015.0127\#. http://doi.org/10.1089/chi.2015.0127.

33. Gevers DWM, Kremers SPJ, De Vries NK, Van Assema P. Patterns of Food Parenting Practices and Children's Intake of Energy-Dense Snack Foods. Nutrients [Internet]. 2015 [citado 2018 Ago 21]; 7(6): 4093-4106. Disponible en: https://www.mdpi.com/2072-6643/7/6/4093/pdf. https://doi.org/10.3390/nu7064093

34. Aquino-Rojas EE. Tácticas para la prevención de la desnutrición en niños menores de 6 meses a través de la concientización de la lactancia materna exclusiva en la población que asiste al Centro de Salud Universitario Norte de la ciudad de Santa Cruz. Rev Cient Salud UNITEPC[Internet]. 2018 [citado 2018 Ago 18]; 4(1): 21-29. Disponible en: https://investigacion.unitepc.edu.bo/revista/index.php/revista-unitepc/article/view/30/49.

35. Hernández-Rincón EH, Arias-Villate SC, Gómez-López MT, León-Pachón LE, Martínez-Ceballos MA, Chaar-Hernández AJ et al. Actividad física en preescolares desde atención primaria orientada a la comunidad, en un municipio de Colombia. Rev Cubana Pediatr [Internet]. 2018 Jun [citado 2018 Ago 18]; 90(2): 201-212. Disponible en: http://scielo.sld.cu/scielo.php?script=sci_arttext\&pid=S0034-75312018000200002\&lng=es.

36. Lázaro-Cuesta L, Rearte A, Rodríguez S, Niglia M, Scipioni H, Rodríguez D, Salinas R, et al. Estado nutricional antropométrico, bioquímico e ingesta alimentaria en niños escolares de 6 a 14 años, General Pueyrredón, Buenos Aires, Argentina. Archiv argent pediat [Internet]. 2018 [citado 2018 Sep 13]; 116(1): e34-e46. Disponible en: https:/www.sap.org.ar/docs/publicaciones/archivosarg/2018/v116n1a15.pdf.

https://dx.doi.org/10.5546/aap.2018.e34. 
37. Ceres-Mattos DL, Menezes-Santos LL, Pereira-da Silva B, Anunciação PC, Gonçalves-Alfena RC, Castro-Franceschini SC, Duarte-Martino HS, Pinheiro-Sant'Ana HM. Impact of rice fortified with iron, zinc, thiamine and folic acid on laboratory measurements of nutritional status of preschool children. Ciênc. saúde coletiva [Internet]. 2017 Feb [citado 2018 Jul 29]; 22(2): 583-592. Disponible en:

http://www.scielo.br/scielo.php?script=sci_arttext\&pi$\mathrm{d}=\mathrm{S} 1413-81232017000200583 \& \operatorname{lng}=\mathrm{en}$. http://dx.doi.org/10.1590/1413-81232017222.17322016. 38. Redsell SA, Edmonds B, Swift JA, Siriwardena AN, Weng S, Nathan D, et al. Systematic review of randomised controlled trials of interventions that aim to reduce the risk, either directly or in directly, of overweight and obesity in infancy and early childhood. Matern Child Nutricion [Internet]. 2016 [citado 2018 Ago 23]; 12(1): 24-38. Disponible en: https://onlinelibrary.wiley.com/doi/epdf/10.1111/mcn.12184. doi: 10.1111/mcn.12184.

39. Pirán-Arce MF, Aballay LR, Leporati JL, Navarro A, Forneris M. Niveles de hierro en sangre según adherencia a la dieta libre de gluten en niños celiacos de edad escolar. Nutric Hospit [Internet]. 2018 [citado 2018 Dic 21]; 35(1): 25-32. Disponible en: https://www.nutricionhospitalaria.org/index.php/articles/00919/show\#!. https://dx.doi.org/10.20960/nh.919.

40. Sachdev HPS, Gera T, Nestel P. Effect of iron supplementation on mental and motor development in children: systematic review of randomised controlled trials. Public Healt [Internet]. 2005 [citado 2018 Dic 23]; 8(2): 117-132. Disponible en: https:/www.cambridge.org/core/ser-

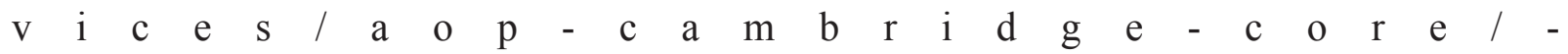
content/view/C670976B3D6735E0EE1980B98815D8AF/S1368980005000194a.pdf/effect_of _iron_supplementation_on_mental_and_motor_development_in_children_systematic_review_ of_randomised_controlled_trials.pdf. https://doi.org/10.1079/PHN2004677.

41. Ballonga-Paretas C, López-Toledo S, Echevarría-Pérez P, Vidal-Corrons Ó, Canals-Sans J, Arija-Val V. Estado nutricional de los escolares de una zona rural de extrema pobreza de Ccorca, Perú. Proyecto INCOS. ALAN [Internet]. 2017 Mar [citado 2018 Oct 19]; 67(1): 23-31. Disponible $\quad$ en: http://ve.scielo.org/scielo.php?script=sci_arttext\&pi$\mathrm{d}=\mathrm{S} 0004-06222017000100004 \& \operatorname{lng}=\mathrm{es}$.

42. Osorio AM, Romero GA, Harold-Bonilla H, Aguado LF. Contexto socioeconómico de la comunidad y desnutrición crónica infantil en Colombia. Rev Sal Públ [Internet]. 2018 [citado 2018 Dic 18]; (52): 1-12. Disponible en: http://www.scielo.br/pdf/rsp/v52/es_0034-8910-rsp-S1518-52-87872018052000394.pdf.

https://doi.org/10.11606/S1518-8787.2018052000394.

43. López-Rodríguez G, Galván M, Fuentes-Galicia SJ. Indicadores de síndrome metabólico en escolares mexicanos con talla baja, sobrepeso u obesidad. ALAN [Internet]. 2016 Dic [citado 2018 Ago 07]; 66(4): 309-317. Disponible en: http://ve.scielo.org/scielo.php?script=sci_arttext\&pid=S0004-06222016000400007\&lng=es. 
44. Macollunco-Flores P, Ponce-Pardo J, Inocente-Camones M. Programas nacionales para la prevención y tratamiento de anemia ferropénica en los países de Sudamérica. Sal Públ Méx [Internet]. 2018 [citado 2018 Dic 5]; 60(4): 386-387. Disponible en: http://saludpublica.mx/index.php/spm/article/view/9237. http://dx.doi.org/10.21149/9237.

45. Somsri P, Satheannoppakao W, Tipayamongkholgul M, Vatanasomboon P, Kasemsup R. A Cosmetic Content-Based Nutrition Education Program Improves Fruit and Vegetable Consumption Among Grade 11 Thai Students. J Nutr Educ and Behav [Internet]. 2016 [citado 2018 Jul 17]; 48(3): 190-198.e1. Disponible en: https://www.jneb.org/article/S1499-4046(15)00735-6/pdf. https://doi.org/10.1016/j.jneb.2015.11.002.

46. Miranda M, Olivares GM, Durán-Pérez J, Pizarro AF. Anemia prevalence and nutritional status in children of a suburban area of Sucre, Bolivia. Rev chil nutr [Internet]. 2015 Dec [citado 2018 Jul 10]; 42(4): 324-327. Disponible en: https://scielo.conicyt.cl/scielo.php?script=sci_arttext\&pid=S0717-75182015000400001\&lng=en.

http://dx.doi.or$\mathrm{g} / 10.4067 / \mathrm{S} 0717-75182015000400001$.

47. Calderón-Vallejo C. Desnutrición intra-hospitalaria en niños del Hospital Pediátrico Alfonso Villagómez de la ciudad de Riobamba. Rev CienciAméRica [Internet]. 2017 [citado 2018 Jul 14]; 6(2): 93-96. Disponible en: http:/www.cienciamerica.us/openjournal/index.php/uti/article/view/124.

48. Lo Brutto G, Aceves-López L. Cuando el destino nos alcanza. La relación entre movimientos sociales y gobiernos latinoamericanos de izquierda. Tla-melaua [Internet]. 2017 [citado 2018 Ago 18]; 11(43): 156-177. Disponible en: http://www.scielo.org.mx/scielo.php?script=sci_arttext\&pid=S1870-69162017000300156\&lng=es\&tlng=es.

49. Soncco-Sucapuca M, Brousett-Minaya MA, Pumacahua-Ramos A. Impacto de un programa educativo incluyendo un pan fortificado para reducir los niveles de anemia en niños escolares de Yocará, Puno -Perú. Rev Invest Altoandinas [Internet]. 2018 [citado 2018 Oct 06]; 20(1): 73-84. Disponible en: http://huajsapata.unap.edu.pe/ria/index.php/ria/article/view/331. https://dx.doi.org/10.18271/ria.2018.331.

Recibido: 12 de octubre de 2018

Aprobado: 22 de noviembre de 2018 